A C A S E

oF

\title{
UNILATERAL ATROPHY OF THE TONGUE.
}

\author{
BY \\ WM. FAIRLIE CLARKE, M.A. OxoN., F.R.C.S., \\ ASSISTANT-SURGEON TO CHARING CROSS HOSPITAL, AND TO THE CENTRAL \\ LONDON OPHTHALMIC HOSPITAL.
}

Received November 14th-Read November 28 th, 1871.

LAsT June I had an opportunity of seeing an example of unilateral atrophy of the tongue, and as this is a very rare affection, it may be interesting to the Society if $I$ relate the history of the patient.

In order to add to the value of this communication, as well as to throw light upon the subject, $I$ have placed by the side of my own case two others-the only two that I have been able to find noted in medical literature. The one has been put upon record by Baron Dupuytren, the other by Sir James Paget.

The following are the particulars of the case that I saw, so far as I have been able to obtain them; and though the history is not so complete as I could wish, still even an imperfect record of such a rare affection may not be without its value : 
Mrs. H-, æt. 45, the wife of an oilman in the north of London, became aware of a tumour in her right breast in the spring of 1869. Up to this time her health had been good. The menses had ceased two years before. On the 14th of February, 1870, she consulted Mr. Hume, of Devonshire Street, Islington Green, about the tumour in her breast, and he recommended that it should be removed without delay, as it had all the appearance of a malignant growth. The operation was performed on the 16th by Mr. Barwell. The wound healed slowly, but satisfactorily.

On April 15th the patient complained of cough and slight dyspnoa-the latter noticeable only after exercise. Under treatment the cough soon disappeared, but the dyspnœa continued.

On October 3rd Mr. Hume was called suddenly, and found the patient suffering from a deep-seated pain on the right side of the head, of a periodic character, returning each night between 1 and 2 a.m., and rendering her for some hours incoherent and unmanageable. Many remedies were prescribed, but the only thing which gave her any relief was morphia in grain doses. These attacks were accompanied by great turgesence of the vessels on the right side of the neck -a symptom which was partially relieved by the application of leeches to the upper part of the sternum. Subsequently tonics were prescribed, and by the 4th of December the patient was so much better as to require no further attendance. It was at this time that the atrophy of the right side of the tongue was first noticed, though it was not then so marked as it afterwards became.

On March the 29th, 1871, Mr. Hume was again urgently required to attend, and found the patient suffering from all the former symptoms, but in an aggravated degree; and, in addition, there was an alarming dysphagia, together with paroxysms of suffocative cough, which recurred about three times in the twenty-four hours. On being asked to protrude the tongue she always appeared unable to do so at first, and when the request was repeated would reply, "Wait a minute." Then, after a few moments' deliberation, she would put it 
out very slowly. The tongue was crimped and puckered along its whole right side from base to apex, but these appearances were most marked in the anterior two thirds; and an actual loss of substance had taken place, so that this half of the organ seemed small and wasted. The loss of substance was bounded exactly by the median raphé, and the contrast between the plumpness of the left side and the shrivelled aspect of the right was very striking. The tongue was soft

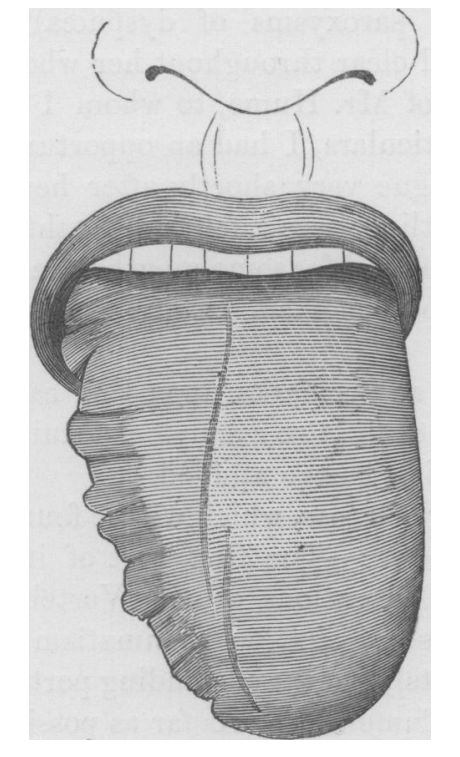

UNILATERAL ATROPHY OF THE TONGUE.

throughout; there were no hard nodules in it. No alteration was noticed either in the common sensation of the organ or in its special sense of taste. When the tongue was protruded there was no deviation to either side. Articulation was slow and difficult. The patient complained of great pain on the right side of the head and along the right side of the neck. There was some fulness on this side of the neck, but no abnormal growth could be felt and no enlarged glands were apparent. When the patient was asleep her breathing 
was stertorous. The chest sounds were normal, but the thorax could not be freely expanded. Along with these symptoms there was general prostration and cachexia.

From this date the patient's condition gradually became worse. The paroxysms of dyspnœa became more frequent and more severe, and the 7th of June, 1871, in one of these paroxysms, she died.

At no time during her illness had she any paralysis of the extremities, and (with the exception of the delirium which accompanied the paroxysms of dyspnœa) her intellectual faculties remained clear throughout her whole illness.

By the favour of Mr. Hume, to whom I am indebted for the foregoing particulars, I had an opportunity of examining the patient's tongue very shortly after her death; and the accompanying outline gives a fair and truthful representation of it, as it had appeared for some months previously. I regret to say that no post-mortem examination of the body was allowed.

Before I offer any remarks upon this case it will be well that I should quote the two examples of unilateral atrophy of the tongue to which I have alluded.

Baron Dupuytren's case, which will be found in the 'Leçons Orales,' is as follows :- The first part of it is given in the lecture upon "Dislocations of the Vertebræ" (for it was regarded as a case of chronic rheumatism of the occipitovertebral ligaments), and the concluding portion in the lecture upon " Hydatid Tumours." As far as possible I have related it in the author's own words.

A man, æt. 30, a weaver by trade, was admitted into the Hôtel-Dieu under the care of M. Dupuytren, about 1830.

Three years before the patient began to suffer from severe pains in the left side and back of the head. These pains prevented him from moving it, and deprived him entirely of sleep. After five or six days they changed their place and became fixed in the upper part of the left side of the neck. From this time they were less violent, but movement still continued impossible.

\footnotetext{
1 'Leçons Orales,' i, 493, and iii, 361 (first edition).
} 
"These symptoms were soon followed by a slight difficulty in speaking, which increased gradually, so that at the end of two months the patient could no longer make himself heard. He said that the air passed in a whistling manner by the left side of his tongue, and that when he wished to pronounce je he said $z e$.

"Some pain was felt at the angle of the lower jaw and in the left cheek, but there was no paralysis of the muscles of this region.

"Another still more extraordinary symptom manifested itself. The left side of the tongue began to diminish in size, and this to such a degree that it became entirely atrophied. This portion of the organ seemed to consist of nothing but folded membranes, which could be rubbed together without feeling any muscles between them. It was like an empty purse. The mucous membrane remained entire, but the muscular tissue beneath it had disappeared. When the tongue was protruded the right side was seen to be well nourished. The atrophy of the left side was greatest in the anterior and middle portions, and was least at the base.

"It also seemed that the right side had acquired more strength. When the tongue was put out this side was curved, either because of its superior strength or because it was no longer supported by the left side. During the first months the patient had been entirely deprived of speech, but when he was examined by M. Dupuytren he spoke as if his tongue were not in the slightest degree wasted. He expressed himself clearly and distinctly." "But to be able to articulate sounds he had needed practice and careful study. The favorable result was due rather to exercise than to any diminution of the disease."

During the time that the patient remained in the HôtelDieu M. Dupuytren made careful observations with regard to his sense of taste, and ascertained that it was not at all impaired.

His intellectual faculties were never in any degree affected, nor had he any paralysis of the extremities.

So much for the state of the patient at the time he was 
first examined. At a later date M. Dupuytren gives us the rest of his history.

"When that man left the Hôtel-Dieu he had paralysis of the left side of the tongue which had determined the atrophy of that part; but he preserved the sense of taste, which made me think that the lesion bore upon the great hypoglossal nerve. Nearly two years passed without my hearing anything of him, when $I$ learnt that he had gone to die at the hospital Cochin. Dr. Gendrin, who was kindly desirous of giving me information about the patient, told me that he preserved his intellectual faculties almost up to the last moments of his life. The paralysis of the left side of the tongue and the atrophy was even more marked than at the time he left the Hôtel-Dieu; the sense of taste had slightly diminished on the affected side, but nevertheless it remained. The patient said that his generative functions were very weak. Some days before his death, symptoms of compression showed themselves and the patient succumbed. At the autopsy, which was made with care, a great deal of serosity was found in the ventricles; but what is the most remarkable is that a large number of hydatids were discovered at the base of the cerebellum ; one of these hydatids had introduced itself into the anterior condyloid foramen, and compressed, in the most evident manner, the great hypoglossal nerve."

The following is Sir James Paget's case, which will be found in the 3rd vol. of the Clinical Society's Reports :

" $\mathrm{H}$. G-, 27 years old, a dairyman, was admitted into St. Bartholomew's Hospital on June 10th, 1869.

"Six years before admission he fell heavily on the back of his head. He was not stunned, but the injury was followed by constant pain and stiffness about the back of the head and neck. Ten months after the fall, an abscess formed and discharged at the back of the head. The stiffness and very free discharge had existed ever since. A fortnight before admission he noticed that his tongue, when protruded, was directed towards the right. His general health had never been severely disturbed. 
" He was a healthy-looking man, except from the stiffness of his neck, which was nearly complete. In the scalp, over the occipital bone, numerous sinuses led to dead and bare bone, and to cavities containing pus, some of which extended over the upper cervical vertebræ. The integuments were thick and tough, infiltrated, and, where not undermined with pus, adherent to the subjacent textures.

"The speech was thick and slow. The muscles of the right side of the tongue were exceedingly wasted. This half of the tongue looked less than half as large as the left; it was collapsed, wrinkled, soft, sunken, like a tongue with wasting palsy, and the muscles of its left side alone appeared to act. Its sensations were unimpaired."

"I removed," says Sir James Paget, "all the dead portions of bone that I could find. They were derived from the posterior and lower part of the occipital bone, some including the whole thickness of the bone, so that the dura mater below the lateral sinuses was exposed, in a space of rather more than an inch in diameter. One of the portions of dead bone included the posterior third of the border of its foramen magnum; another contained the right posterior condyloid foramen."

"The patient recovered from the operation without hindrance, and in a month nearly all the sinuses were healed, and no dead bone could be felt. A few days after the operation the wasted part of the tongue began to grow larger, and within a month it had nearly regained its former size and muscular power.

"Six months after the operation the patient was in good health. Nearly all the sinuses were healed. The tongue was still inferior in both texture and size on its right side, but the difference was comparatively slight; and when put out the tip went but little over to the right side. The rotatory movements of the head were nearly free; the bending movements much less so."

"The case," adds Sir James Paget, "scarcely needs comment. It is very rare as an instance of necrosis involving the border of the foramen magnum, and not attended by voL. Lv. 
grave cerebral or spinal disturbance. But its chief interest is in the wasting of the muscles of half the tongue-a wasting so rapid that it may be ascribed to some morbid condition of the hypoglossal nerve or of filaments enclosed in it, and as rapidly recovered from when the morbid condition was brought to an end by the removal of the dead bone."

Remarks.-In these two cases the cause of the unilateral atrophy was ascertained beyond a doubt. In Dupuytren's case it was demonstrated by post-mortem examination; in Paget's case it was proved by the history of the illness and by the fact that recovery took place when the dead bone, which gave rise to it, was removed. But in my case the evidence is not so complete. Unhappily no autopsy could be obtained. We can, therefore, only infer the cause of the wasting. However, looking at all the circumstances, I think there is good reason to suppose that it depended upon the involvement of the right hypoglossal nerve in a secondary cancerous growth, either inside the cranium or at the upper part of the neck. The facts which lead me to this conclusion are-the existence of an undoubted cancerous tumour, which was removed sixteen months before death, after it had been growing about a year; the general cachexia; the intense and deep-seated pain on the right side of the head and neck; the fulness and turgescence of the vessels on the right side of the neck, denoting an obstruction to the circulation; the frequent attacks of dyspnœa and dysphagia, which probably depended on pressure upon the pneumo-gastric and glossopharyngeal nerves. These symptoms, together with the absence of paralysis of the extremities and the clearness of the intellect, seem to indicate that the disease was not in the brain or medulla oblongata, and make it probable that there must have been an adventitious growth pressing upon, and destroying the function of, the right hypoglossal nerve, and implicating, more or less, the pneumo-gastric and the glossopharyngeal nerves as well. Such a tumour might be situated either inside the cranium, so as to bear upon the 
roots of these nerves where they come off in close juxtaposition from the medulla oblongata; or just outside the cranium, so as to involve the hypoglossal nerve where it emerges from the anterior condyloid foramen, and where it is intimately associated with the glosso-pharyngeal and vagus nerves, or possibly in both these situations at the same time; indeed, the analogy of the cases I have quoted would lead one to say that the hypoglossal nerve may not improbably have been compressed in the anterior condyloid foramen itself.

I am well aware that the evidence $I$ have adduced in favour of a secondary cancerous tumour is not conclusive. It falls far short of the demonstration which an autopsy would have afforded. Still, though the cause of the unilateral atrophy must be admitted to be unproven, the fact of the remarkable appearance of the tongue remains; and that is the point to which I desire to direct attention, and which forms the proper subject of this communication.

But, it may be asked, what reason have we to believe that this wasted condition of the tongue depends upon a lesion of the ninth nerve? Is there any direct proof to show that if the right hypoglossal nerve were thus compressed or destroyed by an adventitious growth, so that its functions were suspended, we should find the right half of the tongue atrophied? To this it may be answered that the ninth nerve has been frequently divided, and that the result has been to produce atrophy of the tongue upon the same side. It is a nerve which may easily be reached underneath the jaw, where it lies between the tendon of the digastric and the hyoid bone; and it has been often made the subject of experiment. Romberg in his work on the 'Nervous Diseases of Man,'* in speaking of the hypoglossal nerve, says, "Atrophy follows paralysis in the muscles of the tongue more rapidly than in other paralysed muscles. Bidder always found that the nutrition of the tongue was impaired during the first week after the division of the hypoglossus, in living dogs, though in no case a vessel of any magnitude had been injured.

\footnotetext{
* Sydenham Society's ‘Transactions,' vol. ii, 304.
} 
Large and deep transverse rugæ were visible on the surface of the tongue, as if the envelope of the organ had become too loose for its fleshy contents ; a manifest difference was perceptible between the two halves."

This affection, then, of the tongue is an example of the large class of muscular atrophies which depend upon the lesion of nerves, and the impairment of nervous functions. The hypoglossal nerve being injured there follows a paralysis of the corresponding side of the tongue, and the muscles which are thus deprived of their contractile power rapidly waste, notwithstanding that they may be kept in constant movement by the muscles of the unparalysed side. But it is the rarity with which this disease occurs, the complex nature of the organ which it affects, the nicety with which the atrophy is bounded by the median raphé, and the fact that it depends, not upon central but upon intermediate disease - disease which in some cases at least is capable of removal - which gives it a peculiar importance and interest.

Appendix.-In order to test for myself the effect of a lesion of the ninth nerve, I performed the following experiment upon a rabbit. On the 25th of October (1871), with the assistance of Dr. William Ogle, I divided the right hypo. glossal nerve, and removed a piece about a quarter of an inch in length.

The nerve was reached with very little difficulty, beneath the angle of the jaw, where it lies above the hyoid bone and below the tendon of the digastric muscle. The portion removed was situated in front of the descendens noni and thyro-hyoid branches, but behind those which are distributed to the stylo-glossus, the genio-hyoid, the hyo-glossus, the genio-hyo-glossus and the intrinsic muscles of the tongue.

Immediately after the operation, when the tongue was protruded, it was turned strongly towards the right, as if the animal were licking the right corner of its mouth. The wound completely healed in a few days. The rabbit was examined from time to time, and on each occasion its tongue was invariably put out towards the right side. 
Yesterday, November 27th, that is to say, thirty-four days after the operation, the rabbit was killed, and the accompanying preparation shows the state of the hypoglossal nerve and of the tongue. The skin, including the cicatrix, could be raised with ease. There was no matting of the subjacent tissues, so that the different parts at the seat of the operation could be clearly discerned. The divided nerve was found to have united by what appears to be a soft semitransparent substance, which is pink and highly vascular, and twice as thick as the ordinary calibre of the nerve. No very marked change is apparent in the tongue, but the middle third, or perhaps rather the posterior half, is somewhat wasted and flattened.

Postscript.-When the rabbit's tongue had been hardened in spirit, and a transverse section of it was made, the atrophy of the right side was very marked-indeed this side of the organ was not above half the size of its fellow.

In the discussion which followed the reading of this paper other recorded cases of a somewhat similar kind were mentioned. (1.) Mr. Holthouse, in his work 'On Squinting and Paralytic Affections of the Eye,' narrates a case of "Paralysis of the sixth and ninth cerebral nerves of the left side, of syphilitic origin, producing inversion of the left eye and wasting of the left half of the tongue." (2.) Dr. Hughlings Jackson relates two cases in which, among other symptoms, the ninth nerve was paralysed. 'London Hospital Reports,' vol. i (1864), p. 361, vol. iv (1867), p. 315. (3.) In the 'Encyclopæedia of Anatomy and Physiology' (art. Tongue), Dr. Hyde Salter alludes to a case which occurred in the practice of Dr. W. Budd. A man was stabbed in the neck-the wound dividing the external carotid artery and the hypoglossal nerve on the left side. The artery was secured, and the patient recovered with palsy of the left side of the tongue. At the end of some weeks that half of the tongue was much wasted, and all the movements of the organ were performed by the other half. The atrophy was confined to the muscular element of the organ; taste and 
touch remaining unimpaired. (4.) A case, which is very analogous to that which forms the subject of this paper, is related by Dr. Habershon in the 'Medical Times and Gazette' for February 9th, 1867. 